\title{
Correlation between synovial neopterin and inflammatory activity in rheumatoid arthritis*
}

\author{
ANDRES KRAUSE, HUBERTUS PROTZ, AND KLAUS M GOEBEL \\ From the Division of Rheumatology, Department of Medicine, University Hospital Marburg, West Germany
}

SUMMARY According to recent investigations neopterin (a pyrazinopyrimidine derivative) is a biochemical marker that reflects the activity of the proinflammatory immunocellular system of the synovial tissue in rheumatoid arthritis (RA). Interferon gamma, derived from antigen ${ }_{\infty}^{\infty}$ activated $\mathrm{T}$ lymphocytes, stimulates macrophages to synthesise and release neopterin into the $\sigma^{\circ}$ culture supernatant in vitro. To extend this in vitro model to a clinical level a sensitive newo radioimmunoassay technique was used to measure neopterin concentrations in the synovial fluid은 (SF) of 17 patients with active RA, nine with osteoarthritis, and six with acute gout, and in that of $\rightarrow$ 12 controls undergoing meniscectomy. The SF neopterin concentrations were significantly higher: in patients with RA than in the other groups of patients, particularly the controls. Multivariant analysis showed that SF neopterin concentrations correspond better with the systemic $\vec{\oplus}$ inflammatory activity of RA than with the local disease activity of the knee joints. Thus the study strengthens the hypothesis that neopterin reflects the essential role of the activated immunocellular reaction in the pathogenesis of RA.

Neopterin (a pyrazinopyrimidine compound) is synthesised from guanosine triphosphate by macrophages. ${ }^{1}$ In vitro, it has been shown that its release is stimulated by interferon gamma derived from antigen activated T lymphocytes. ${ }^{2}$ Thus neopterin is a specific marker for the activation of the cellular immune system. Its specific role in the cellular immune response is still unknown, however, as it is only an intermediate product in the synthesis of the cofactor biopterin. As mononuclear cells accumulate in the synovia of inflamed joints in patients with rheumatoid arthritis (RA) ${ }^{3}$ and as the pathogenesis of RA depends on the central immunoregulating effect of antigen activated $T$ lymphocytes, ${ }^{4}$ neopterin should be released into the synovial fluid (SF) in such patients. Therefore, in an attempt to extend the in vitro experiments to a clinical level we made a comparative study of the diagnostic significance of SF neopterin in patients with RA and with other rheumatoid diseases and tried to correlate the SF neopterin concentrations in

Accepted for publication 17 January 1989.

Correspondence to Dr Andres Krause, Department of Medicine, University Hospital, PO Box 2360, D-3550 Marburg, W Germany. *Presented in part at the annual meeting of the American Federation for Clinical Research, New York, USA, 1988. (Clin Res 1988;36:798A).
RA with the degree of systemic and local inflammatory activity.

Patients and methods

PATIENTS, CLINICAL ASSESSMENT, AND SF? COLLECTION

From three groups of ambulatory outpatients with 3 persistent knee effusion, who regularly attended the rheumatic disease service of the University Hospital of Marburg, West Germany, we selected 32, who, according to the 1987 modified American Rheumat- ism Association criteria ${ }^{5}$ had classical or definite RA $(n=17)$, osteoarthritis $(n=9)$, or acute gout $(n=6)$. Table 1 shows their demographic and clinical $/$ characteristics. All patients were receiving treat- $N$ ment with non-steroidal anti-inflammatory agents, N but not prednisolone, and six of 17 patients with $\mathrm{RA}_{\sigma}^{\mathrm{\omega}}$ were receiving gold treatment. The criteria for exclusion included a history of RA of less than two months; associated psoriasis, seronegative spondylo- $\cong$ arthropathy, or Reiter's disease; and recent or anticipated treatment with systemically or intraarticularly administered corticosteroids. All patients with osteoarthritis had radiologically proved degen- $\frac{?}{0}$ erative joint disease and at least one of the following $\varrho$ criteria of disease activity: swelling, pain withe 
motion, and pain at rest. To obtain normal mean control values we also measured the neopterin concentrations in the non-inflammatory SFs of 12 patients undergoing meniscectomy. The grading of RA in individual patients was performed according to radiographic criteria of Steinbrocker, modified by Larsen et al. ${ }^{6}$ Six patients had stage I disease, four had stage II, five had stage III, and two had stage IV. The local disease activity of RA was clinically assessed on the basis of the following subjective and objective findings and scored from 0 to 5 : pain at rest or with motion (from 'no pain' to 'most severe'), the difference in joint circumference (from $0.5 \mathrm{~cm}$ to $4.0 \mathrm{~cm}$ in $0.7 \mathrm{~cm}$ steps), and difference in skin temperature of the knee joint (from $0.5^{\circ} \mathrm{C}$ to $2.4^{\circ} \mathrm{C}$ in $0.5^{\circ} \mathrm{C}$ steps). ${ }^{78}$ The total volume of knee joint $\mathrm{SF}$ and the echogenic areas of the pannus and the inflamed knee joint capsule were assessed with ultrasonography. ${ }^{8}$ Ultrasonographic scans were made transversely and longitudinally on both knees using a commercial contact high resolution real time scanner, with a $7.5 \mathrm{MHz}$ transducer. The sodium pertechnetate ${ }^{99 \mathrm{~m}} \mathrm{Tc}$ uptake in the knee joint ${ }^{99 \mathrm{~m}} \mathrm{Tc}$ index) was measured as a percentage of the total injected activity, also according to a previously reported method. ${ }^{9}$ Radiographs of the knee joints were scored from 0 to 3 according to the degree of destruction, as in a previous study. ${ }^{10}$ Indicators of systemic inflammatory disease activity were scored from 0 to 5 and included the Ritchie index, ${ }^{11}$ the degree of anaemia and thrombocytosis, and the erythrocyte sedimentation rate. Finally, the individual total index was calculated by adding the scores of all subjective and objective tests and then dividing them by the number of tests to give a final score that indicated mild (class I), moderate (class II), or severe (class III) local or systemic disease activity, according to previously described methods. 812

\section{LABORATORY TESTS ON SF}

As much SF as possible was aspirated and then immediately analysed for total and differential leucocyte counts. Biochemical tests and immunological studies included total protein, $\mathrm{C}$ reactive protein, and immune complex determinations, performed by immunoturbidimetric assay ${ }^{13}$ using an automated micropipette nephelometer system (Beckman Immunochemistry Systems, Munich). The prostaglandin $\mathrm{E}_{2}$ concentration was measured by radioimmunoassay. ${ }^{14}$ The neopterin concentration was determined with a new, highly sensitive radioimmunoassay, according to a previously reported method, ${ }^{15}$ in which aliquots $(150 \mu \mathrm{l})$ of the unextracted samples are analysed in triplicate at a 1:10 dilution in the standard diluent of the test:
Table 1 Patients' demographic and clinical characteristics

\begin{tabular}{|c|c|c|c|}
\hline & $\begin{array}{l}R A^{*} \\
(n=17)\end{array}$ & $\begin{array}{l}\text { Osteoarthritis } \\
(n=9)\end{array}$ & $\begin{array}{l}\text { Gout } \\
(n=6)\end{array}$ \\
\hline Women & 11 & 2 & 0 \\
\hline Men & 6 & 7 & 6 \\
\hline Age (years) $\dagger$ & $48 \cdot 1(2 \cdot 7)$ & $63 \cdot 0(2 \cdot 3)$ & $41 \cdot 8(2 \cdot 3)$ \\
\hline $\begin{array}{l}\text { Duration of disease } \\
\text { (years) } \dagger\end{array}$ & $6 \cdot 5(1 \cdot 5)$ & $12 \cdot 5(6 \cdot 5)$ & $0.29(0.06)$ \\
\hline Treatment & & & \\
\hline NSAIDs* & 17 & 9 & 6 \\
\hline Gold & 6 & - & - \\
\hline Steroids & - & - & - \\
\hline
\end{tabular}

${ }^{*} \mathrm{RA}=$ rheumatoid arthritis, NSAIDs=non-steroidal anti-inflammatory drugs.

†Values are means (SEM).

details of the binding affinity and the immunological specificity of antineopterin antibody have been described elsewhere. ${ }^{15}$

Briefly, before radioimmunoassay of the SF neopterin each sample was generally submitted to iodine oxidation in $0.2 \mathrm{M} \mathrm{HCl}$ to obtain the total concentration of neopterin and its didydro and tetrahydro derivatives. The radioimmunoassay used was highly specific for D-erythroneopterin with cross reacting antibodies to various structurally related pteridines. Thus $N^{2}$-carboxypropylneopterin was used as actual hapten, with its radioiodinated tyramine derivative as the tracer, a D-erythroneopterin equivalent was read off from a standard curve, and then the value was divided by the amount of each pterin, multiplied by 100 to obtain the percentage of the cross reaction. For radioimmunoassay of the SF neopterin a mixture of $150 \mu \mathrm{l}$ of sample or standard compound in $0.02 \mathrm{M}$ phosphate buffer $\mathrm{pH} 7 \cdot 5$ containing $0.1 \%$ bovine serum albumin, $100 \mu \mathrm{l}$ of 8000 -fold diluted antiserum, and $100 \mu \mathrm{l}$ of the above buffer was incubated at $37^{\circ} \mathrm{C}$ for 30 minutes. A solution of the tracer $(100 \mu l, 20000$ cpm) was added, and the mixture was kept at $4^{\circ} \mathrm{C}$ for one hour. Antigen-antibody complex was precipitated by a second antibody method ${ }^{15}$ using antirabbit IgG and dextran T-70, and the radioactivity of the precipitate was counted.

With 6000 to 8000 disintegrations per minute of tritiated neopterin (Henning Radiochemical Centre, West Berlin) in a volume of $1.5 \mathrm{ml}$ the lowest detectable concentration of the compound was $20 \mathrm{pg} / \mathrm{ml}$ of SF.

\section{STATISTICAL ANALYSIS}

Sensitivity was defined as the proportion of patients with a given diagnosis in whom the neopterin test was positive, and specificity as the proportion of patients without the diagnosis in whom the test was 


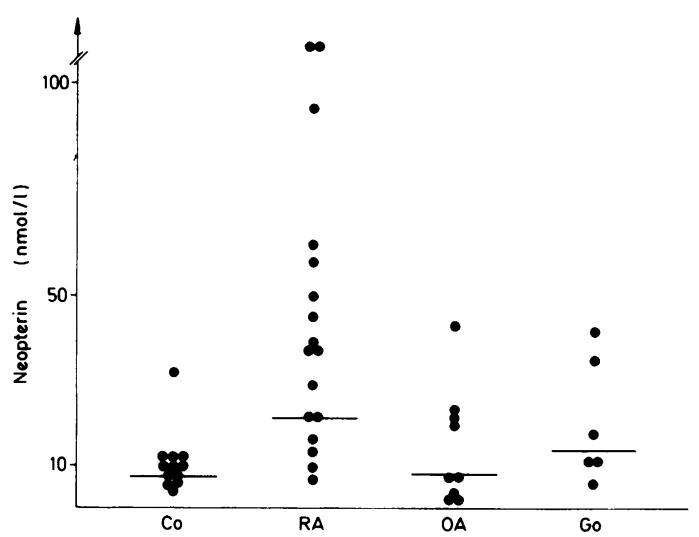

Fig. 1 Neopterin concentrations in synovial fluid of patients with rheumatoid arthritis $(R A)(n=17)$, osteoarthritis $(O A)(n=9)$, and gout $(G o)(n=6)$ and in 12 controls $(\mathrm{Co})$ undergoing meniscectomy. $p<0.001$ for patients with $R A v$ controls; $p<0.05$ for patients with $R A v$ those with osteoarthritis and those with gout.

negative. The results for individual patients (expressed as arithemetic means (SEM)) were compared by analysis of variance using Wilcoxon's signed rank test for paired data. A rank test was applied to the rheumatoid activity index because the paired clinical differences were not normally distributed by Student's $t$ test. Analysis with the FischerYates's correction formula was used for pairs of discrete variables to enable comparison with other data. The significance of all statistical tests was set at the $5 \%$ level $(0.05)$. Stepwise logistic regression was used to determine the simultaneous effect of systemic and local variables on neopterin concentrations. $\overrightarrow{\vec{F}}$ The respective three way contingency tables were $\stackrel{?}{+}$ analysed with a log-linear model in the BMDP4F computer program. ${ }^{16}$

\section{Results}

Neopterin was detected in all SF specimens obtained $\vec{O}$ from patients with rheumatic diseases because of the $\overrightarrow{-}$ high sensitivity of the radioimmunoassay used in this ${ }_{\sigma}^{\omega}$ study. The neopterin concentrations of patients with@ RA were significantly higher than those of controls? $(p<0.001)$, and also higher than those with osteo- $-\frac{\overrightarrow{ }}{\infty}$ arthritis and gout, though to a lesser degree (both $\%$

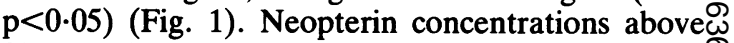
$32 \mathrm{nmol} / \mathrm{l}$ showed a sensitivity of $65 \%$ and $\mathrm{a}_{\circ}$ specificity of $92 \%$ in differentiating RA from osteoarthritis; a neopterin concentration above $\vec{\square}$ $32 \mathrm{nmol} / 1$ had a specificity of $96 \%$. In most patients with RA more than half of the SF cells were $\frac{\bar{N}}{\mathrm{C}}$ polymorphonuclear leucocytes (Table 2). Further, SF neopterin values obtained from patients with RA underwent a rank correlation with diversive SF ${ }^{\circ}$ variables (Table 2). The results of these rank correlations showed: SF neopterin $v$ SF leucocytes (NS); SF neopterin $v$ SF lactate dehydrogenase $\frac{\partial}{0}$ (NS); SF neopterin $v$ SF C reactive protein (NS); but SF neopterin $v$ SF immune complexes $(r=0 \cdot 48, \AA$ $\mathrm{p}<0.02)$. We also obtained a correlation between $S F \overrightarrow{\vec{F}}$ neopterin concentrations and the local and systemic $\stackrel{3}{3}$ disease activity in patients with RA. Local disease $\vec{T}$ activity, as indicated by the ${ }^{99 \mathrm{~m}} \mathrm{Tc}$ indexes and the radiographic scores of the knee joints from which

Table 2 Synovial fluid findings and indicators of local disease activity in controls and patients with rheumatoid arthritis $(R A)$. Values are given as means (SEM)

\begin{tabular}{|c|c|c|c|}
\hline Finding & $\begin{array}{l}\text { Controls } \\
(n=12)\end{array}$ & $\begin{array}{l}\text { Patients } \\
\text { with } R A \\
(n=17)\end{array}$ & p Value \\
\hline Neopterin (nmol/l) & $10 \cdot 3(7 \cdot 3)$ & $41 \quad(9 \cdot 0)$ & $<0.001$ \\
\hline White blood cell count $\left(\times 10^{9} / 1\right)$ & $1.64(0.5)$ & $11 \cdot 3(3 \cdot 2)$ & $<0.01$ \\
\hline Polymorphonuclear leucocytes (\%) & $24 \cdot 4(5 \cdot 2)$ & $59.6(9.4)$ & $<0.01$ \\
\hline Protein $(g / 1)$ & $2.9 \quad(0.6)$ & $5.7 \quad(1.4)$ & $<0.05$ \\
\hline Lactate dehydrogenase (U/l) & $197(38)$ & 304 (51) & $<0.05$ \\
\hline C reactive protein $(\mathrm{mg} / \mathrm{l})$ & $4 \cdot 3(0.7)$ & $12.4(3.2)$ & $<0.01$ \\
\hline Immune complexes (mg/l) & $5 \quad(0.7)$ & 12 (2) & $<0.05$ \\
\hline Prostaglandin $E_{2}(\mu g / 1)$ & $4.47(2.09)$ & $1.01(0 \cdot 46)$ & NS $^{*}$ \\
\hline Local disease activity score & $3.4(0.4)$ & $8.4(1 \cdot 1)$ & $<0.001$ \\
\hline${ }^{99 \mathrm{~m}} \mathrm{Tc}$ index $(\%)$ & $\mathrm{ND}^{*}$ & $12 \cdot 4(4 \cdot 8)$ & - \\
\hline Radiographic score & ND & $2.4 \quad(0.8)$ & - \\
\hline
\end{tabular}

Regression analysis showed no significant correlation $(r=0 \cdot 18)$ between synovial fluid neopterin concentrations and local inflammatory $\stackrel{?}{\Phi}$ activity of the knee joints, as indicated by the local disease activity score, the ${ }^{99 \mathrm{~m}} \mathrm{Tc}$ index, and the radiographic score, considered together. $\varrho$ (See text for explanation of how these were measured.)

${ }^{*} \mathrm{ND}=$ not determined; $\mathrm{NS}=$ not significant. 


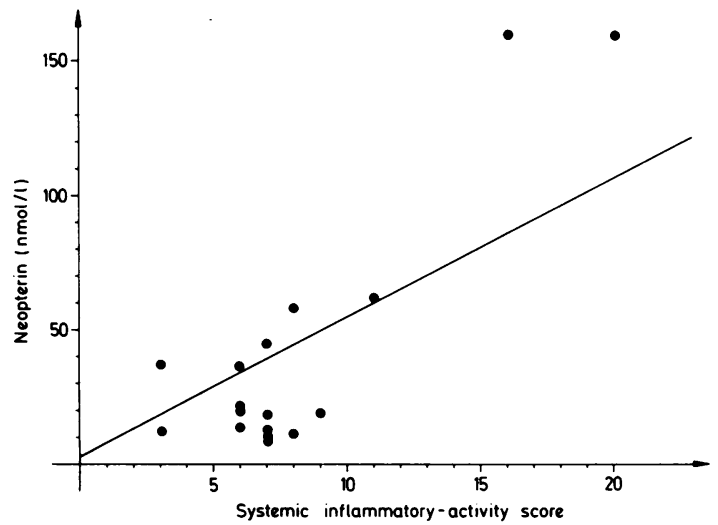

Fig. 2 Multivariant analysis of correlation between neopterin concentrations in synovial fluid and clinical indicators of systemic disease activity in patients with rheumatoid arthritis $(n=17)(p<0.03, r=0.46)$.

the SF had been aspirated, was not significantly correlated with SF neopterin concentrations (Table 2). In contrast, there was a low but significant correlation between neopterin concentrations and the overall severity of systemic inflammatory disease in RA ( $r=0.46, p<0.03)$ (Fig. 2).

\section{Discussion}

The aetiological factor triggering the pathological chain reaction of $\mathrm{RA}$ is still unknown. ${ }^{17}$ The normally thin human synovium undergoes a profound alteration when the aggressive inflammatory process of RA develops. In rheumatoid synovitis three features predominate: inflammation, proliferation, and infiltration. ${ }^{18}$ During the early stages of inflammation in rheumatoid arthritis polymorphonuclear leucocytes are the predominant cells in the $\mathrm{SF}$, and immune complexes, composed of immunoglobulins, complement clearage products, and acute phase proteins-for example, $\mathrm{C}$ reactive protein, can also be detected. ${ }^{18}$ Thus SF analysis has been widely recommended as an important diagnostic procedure in patients with arthritic effusions. ${ }^{19}$ Although repeated SF analysis may provide diagnostic information that is unavailable from history and physical examination and may enable the clinician to reassess various diagnoses, no pathognomonic diagnostic marker for RA has been discovered. ${ }^{18}$ Our results mainly confirm that certain diagnostically non-specific SF findings are typical of RA, as has also been observed for other inflammatory arthritides. Except for neopterin, these findings differed significantly only from those of normal SF specimens obtained from controls undergoing meniscectomy. The important role of the $T$ lymphocyte-macrophage axis in the pathogenesis of RA is widely accepted. ${ }^{20}$ Therefore, we tried to determine whether SF neopterin concentrations are more useful than the conventionally accepted diagnostic indicators. Our most important finding was the high sensitivity and specificity of neopterin concentrations above $32 \mathrm{nmol} / \mathrm{l}$. Even at lower concentrations the comparatively higher neopterin concentrations give a differential diagnostic hint. Multivariant analysis showed that SF neopterin concentrations in patients with RA reflect the degree of systemic inflammatory disease activity more than they reflect the local disease activity of the knee joints. Why are these concentrations not better correlated with the inflammatory activity at the site where the neopterin was synthesised?

The release of neopterin into the SF by locally accumulated macrophages is mainly stimulated by interferon gamma from the systemically activated $\mathrm{T}$ lymphocyte system. ${ }^{21} 22$ This conclusion was confirmed by the results of a previous study ${ }^{23}$ in which urinary neopterin concentrations were found to be greater in patients with RA who had a high degree of systemic disease activity than in those with less systemic activity or with osteoarthritis. The local inflammatory activity, however, is mainly influenced by-and hence corresponds to-the concentrations of prostaglandins, lysosomal enzymes, superoxide radicals released from polymorphonuclear cells, and interleukins, which mediate the production of acute phase proteins. ${ }^{24}$ This lack of correlation between high SF neopterin concentrations and local disease activity might also reflect the fact that a decrease in joint function in RA may be influenced more by erosive bone destruction (as indicated in our study by an increased radiographic score) than by the extent of synovitis and its clinical symptoms. ${ }^{7}$ Neopterin itself is probably only an intermediate product of biopterin synthesis, without any biological activity or any effect on local inflammatory changes. It reflects the activation of the cellular immune system in RA as a systemic inflammatory disorder, and these changes do not necessarily correlate with local inflammatory reactions. To determine the influence of non-steroidal anti-inflammatory drug treatment on neopterin concentrations and on the clinical activity of RA, more follow up data are needed from patients with RA or other autoimmune diseases, such as ulcerative colitis ${ }^{25}$ and Crohn's disease. ${ }^{26}$ As all of our patients with RA received such drug treatment it is possible that it had an effect on the local synthesis and release into SF of prostaglandin $E_{2}$. Non-steroidal anti-inflammatory drug treatment, however, obviously does not affect 
the products of the systemic inflammatory processfor example, $\mathrm{C}$ reactive protein, immunoglobulins, and immune complexes, because they are mediated primarily by interleukins, which remain unaffected by non-steroidal anti-inflammatory agents. ${ }^{24}$ Although the SF neopterin concentrations in our patients with RA correlated significantly with the degree of systemic inflammatory activity, they may not serve as a specific marker for any autoimmune disease because various concomitant infections of malignant disorders may provoke similar $\mathrm{T}$ lymphocyte mediated immune responses, which are invariably associated with an increased release of neopterin by macrophages. ${ }^{22}{ }^{27}$ Thus determination of urinary or SF neopterin concentrations may supplement but should never replace careful clinical evaluation of patients with RA.

\section{References}

1 Huber C, Batchelor J R, Fuchs D, et al. Immune responseassociated production of neopterin. $J$ Exp Med 1984; 160: 310-6.

2 Huber C, Fuchs D, Hausen A, et al. Pteridines as a new marker to detect human $\mathrm{T}$ cells activated by allogeneic or modified self major histocompatibility complex (MHC) determinants. $J$ Immunol 1983; 130: 1047-50.

3 Leeming R J, Blair J A, Melikian V, O'Gorman D J. Biopterin derivates in human body fluids and tissues. J Clin Pathol 1976; 29: 444-51.

4 Janossy G, Duke O, Poulter L W, Panayi G, Bofill M, Goldstein G. Rheumatoid arthritis: a disease of $T$ lymphocyte macrophage immunoregulation. Lancet 1981; ii: 839-42.

5 Arnett F C, Edworthy S M, Bloch D A, et al. The American Rheumatism Association 1987 revised criteria for the classification of rheumatoid arthritis. Arthritis Rheum 1988; 31: 315-24.

6 Larsen A, Dale K, Eek M. Radiographic evaluation of rheumatoid arthritis and related conditions by standard reference films. Acta Radiol [Diagn] (Stockh) 1977; 48: 481-91.

$7 \mathrm{Katz}$ W A. The knee in the diagnosis of rheumatic diseases. In: Katz W A, ed. Rheumatic diseases: diagnosis and management. Philadelphia: Lippincott, 1977: 151-76.

8 Spiegel T M, King W, Weiner S R, Paulus H E. Measuring disease activity: comparison of joint tenderness, swelling, and ultrasonography in rheumatoid arthritis. Arthritis Rheum 1987; 30: $1283-8$.

9 Grimaldi M G. Long term low dose ticlopidine treatment in rheumatoid arthritis: effects on serum sulphydryl levels, technetium index, erythrocyte sedimentation rate, and clinical disease activity. Eur J Clin Pharmacol 1985; 29: 165-8.
10 Hadler N M, Johnson A M, Spitznagel J K, Quinet R J. Protease inhibitor in inflammatory synovial effusions. Ann Rheum Dis 1981; 40: 55-9.

11 Ritchie D M, Boyle J A, McInnes J M, et al. Clinical studies with an articular index for the assessment of joint tenderness in patients with rheumatoid arthritis. $Q J$ Med 1968; 37: 393-406.

12 Farr M, Kendall M J, Young D W, Meynell M J, Hawkins C F. Assessment of rheumatoid activity based on clinical features and blood and synovial fluid analysis. Ann Rheum Dis 1976; 35: 163-7.

13 Goebel K M, Storck U, Krueger K, Schattenkirchner M. Different synovial fluid fibronectin levels in rheumatoid. variants. Klin Wochenschr 1984; 62: 768-72.

14 Tokunaga M, Ohuchi K, Yoshizawa S, Tsurufuji S, Rikimaru A, Wakamatsu E. Change of prostaglandin E levels in joint fluids after treatment with flurbiprofen in patients with rheuma- $\bar{\alpha}$ toid arthritis and osteoarthritis. Ann Rheum Dis 1981; 40: $\frac{\infty}{\infty}$ 462-5.

15 Rokos H, Rokos K. A radioimmunoassay for determination of 90 neopterin. In: Blair J A, ed. Chemistry and biology of pteridines. Berlin: de Gruyter, 1983: 815-9.

16 Dixon W J. BMDP Statistical software. Berkeley, California:응 University of California Press, 1983: 330-4.

17 Anonymous. The viral aetiology of rheumatoid arthritis [Editorial]. Lancet 1984; i: 772-4.

18 Snyderman R. Mechanisms of inflammation and leukocyte chemotaxis in the rheumatic diseases. Med Clin North Am 1986; 70: $217-35$.

19 Eisenberg J M, Schumacher H R, Davidson P K, Kaufmann L. Usefulness of synovial fluid analysis in the evaluation of jointe effusions. Arch Intern Med 1984; 144: 715-9.

20 Dayer J M, Breard J, Chess L, Krane S R. Participation of monocyte-macrophages and lymphocytes in the production of a factor that stimulates collagenase and prostaglandin release by rheumatoid synovial cells. J Clin Invest 1979; 64: 1386-92.

21 Huber Ch, Fuchs D, Niederwieser D, et al. Neopterin: a new biochemical marker for clinical assessment of cell mediated immune response. Klin Wochenschr 1984; 62: 103-13.

22 Ziegler I, Hamm U, Berndt J. Participation of pterins in the control of lymphocyte stimulation and lymphoblast proliferation. Cancer Res 1983; 43: 5356-9.

23 Reibnegger G, Egg D, Fuchs D, et al. Urinary neopterin refleck clinical activity in patients with rheumatoid arthritis. Arthrition Rheum 1986; 29: 1063-70.

24 Wood D D, Ihrie E J, Dinarello C A, Cohen P L. Isolation of an interleukin 1-like factor from human joint effusions. Arthritis. Rheum 1983; 26: 975-83.

25 Niederwieser D, Fuchs D, Hausen A, et al. Neopterin as a new? biochemical marker in the clinical assessment of ulcerative colitis. Immunobiology 1985; 170: 320-6.

26 Prior C, Bollbach R, Fuchs D, et al. Urinary neopterin: a marker of clinical activity in patients with Crohn's disease. Clin Chim Acta 1986; 155: 11-22.

27 Prior C, Fuchs D, Hausen A, et al. Potential of urinary neopterin excretion in differentiating chronic non- $A$, non-B hepatitis from fatty liver. Lancet 1987; ii:1235-7. 